\title{
SOME REMARKS ON L-EQUIVALENCE OF ALGEBRAIC VARIETIES
}

\author{
ALEXANDER I. EFIMOV
}

\begin{abstract}
In this short note we study the questions of (non-)L-equivalence of algebraic varieties, in particular, for abelian varieties and K3 surfaces. We disprove the original version of a conjecture of Huybrechts [9, Conjecture 0.3] stating that isogenous K3 surfaces are L-equivalent. Moreover, we give examples of derived equivalent twisted K3 surfaces, such that the underlying K3 surfaces are not L-equivalent. We also give examples showing that D-equivalent abelian varieties can be non-L-equivalent (the same examples were obtained independently in [12]). This disproves the original version of a conjecture of Kuznetsov and Schinder [14, Conjecture 1.6].

We deduce the statements on (non-)L-equivalence from the very general results on the Grothendieck group of an additive category, whose morphisms are finitely generated abelian groups. In particular, we show that in such a category each stable isomorphism class of objects contains only finitely many isomorphism classes. We also show that a stable isomorphism between two objects $X$ and $Y$ with $\operatorname{End}(X)=\mathbb{Z}$ implies that $X$ and $Y$ are isomorphic.
\end{abstract}

\section{CONTENTS}

1. Introduction

2. On the Grothendieck group of an additive category.

3. L-equivalence of algebraic varieties

References

\section{INTRODUCTION}

In this paper we consider the questions of L-equivalence of complex algebraic varieties, via integral Hodge realization. In particular, we disprove the original versions of conjectures of Huybrechts [9, Conjecture 0.3] and Kuznetsov-Schinder [14, Conjecture 1.6].

We recall the definition of the Grothendieck ring $K_{0}\left(\operatorname{Var}_{\mathbb{C}}\right)$ of varieties. It is generated as an abelian group by the isomorphism classes $[X]$ of reduced separated schemes of finite

Key words and phrases. Grothendieck rings of varieties, Hodge structures, Krull-Schmidt categories. MSC: 18F30, 14C30.

This work is supported by the RSF under a grant 14-50-00005. 
type over $\mathbb{C}$, subject to relation $[X]=[Z]+[U]$, whenever $Z \subset X$ is a closed subset, and $U=X \backslash Z$. The product in $K_{0}\left(\operatorname{Var}_{\mathbb{C}}\right)$ is given by the product of schemes. We denote by $\mathbb{L}$ the class of affine line $\left[\mathbb{A}^{1}\right]$.

Algebraic varieties $X$ and $Y$ are called L-equivalent if the difference $[X]-[Y]$ vanishes in the localization $K_{0}\left(\operatorname{Var}_{\mathbb{C}}\right)\left[\mathbb{L}^{-1}\right]$. In other words, for some $n>0$ we have $([X]-[Y]) \cdot \mathbb{L}^{n}=0$.

The kernel of the homomorphism $K_{0}\left(\operatorname{Var}_{\mathbb{C}}\right) \rightarrow K_{0}\left(\operatorname{Var}_{\mathbb{C}}\right)\left[\mathbb{L}^{-1}\right]$ is hard to control, and finding non-trivial elements in it is an interesting and challenging task. The following conjectures have been made in the original versions of [9] and [14] (both conjectures have been modified in the published versions of the papers).

Conjecture 1.1. [14, original version of Conjecture 1.6] If $X$ and $Y$ are smooth projective simply connected varieties such that $D^{b}(X) \cong D^{b}(Y)$, then $X$ and $Y$ are L-equivalent.

Conjecture 1.2. [9, original version of Conjecture 0.3] Let $S$ and $S^{\prime}$ be complex projective K3 surfaces. The following conditions are equivalent.

(i) $H^{2}(S, \mathbb{Q}) \cong H^{2}\left(S^{\prime}, \mathbb{Q}\right)$ (isomorphism of rational Hodge structures);

(ii) $\mathfrak{h}(S) \cong \mathfrak{h}\left(S^{\prime}\right)$ (isomorphism of rational Chow motives);

(iii) $[S]=\left[S^{\prime}\right]$ in (an appropriate localization of) $K_{0}\left(\operatorname{Var}_{\mathcal{C}}\right)\left[\mathbb{L}^{-1}\right]$.

Below we disprove Conjecture 1.2 (implication (i) $\Rightarrow$ (iii)) in its original version (Corollary 3.5). Moreover, our argument shows that Conjecture 1.2 does not hold even if we localize $K_{0}\left(\operatorname{Var}_{\mathbb{C}}\right)\left[\mathbb{L}^{-1}\right]$ with respect to some set of monic polynomials in $\mathbb{L}$ (say, with respect to $\left(\mathbb{L}^{n}-1\right)$ for all $n>0$, or, equivalently, with respect to $\left.\left[G L_{n}(\mathbb{C})\right], n>0\right)$, see Remark 3.9 .

We give examples of derived equivalent twisted K3 surfaces such that the underlying K3 surfaces are non-L-equivalent (Corollary [3.6, Proposition 3.8).

We also give counterexamples to Conjecture 1.1. (Theorem 3.1). The same examples were independently obtained in [12].

Moreover, we show that both for abelian varieties and K3 surfaces there are only finitely many varieties in each L-equivalence class (Theorems 3.2, 3.4).

Our tool to distinguish L-equivalence classes is the integral Hodge realization. Namely, we use the homomorphism from $K_{0}\left(\operatorname{Var}_{\mathbb{C}}\right)\left[\mathbb{L}^{-1}\right]$ to the Grothendieck group $K_{0}^{\text {add }}\left(\mathrm{HS}_{\mathbb{Z}}\right)$ of the additive category of pure integral Hodge structures, see Section 3 ,

All the statements on non-L-equivalence are deduced from the general results on Grothendieck groups of additive categories whose morphisms are finitely generated abelian groups, see Section 2, In particular, we show that in such an additive category stable isomorphism between $X$ and $Y$ implies an isomorphism if $\operatorname{End}(X)=\mathbb{Z}$ (Theorem 2.3). We also show that in each stable isomorphism class there are only finitely many isomorphism 
classes (Theorem 2.7). Our tools are elementary: reduction to Krull-Schmidt categories (by tensoring with $\mathbb{F}_{p}$ ) and Jordan-Zassenhaus theorem for orders in finite-dimensional semi-simple $\mathbb{Q}$-algebras.

Acknowledgements. I am grateful to Alexander Kuznetsov, Valery Lunts and Dmitri Orlov for useful discussions. I am especially grateful to D. Huybrechts for his comments on the previous version of the paper and for pointing out a minor mistake in the proof of Corollary 3.5. I am also grateful to anonymous referee for useful comments and suggestions, in particular for suggesting Corollary 3.6.

\section{On the Grothendieck Group of an ADditive CAtegory.}

For an additive category $\mathcal{B}$ and an associative unital ring $R$, we denote by $\mathcal{B}_{R}$ the tensor product $\mathcal{B} \otimes_{\mathbb{Z}} R$. This is an additive category with the same objects as $\mathcal{B}$, and the morphisms are given by $\mathcal{B}_{R}(X, Y)=\mathcal{B}(X, Y) \otimes_{\mathbb{Z}} R$. The composition in $\mathcal{B}_{R}$ is induced by the composition in $\mathcal{B}$ and the product in $R$.

We recall that an additive category $\mathcal{B}$ is called a Krull-Schmidt category if each object can be decomposed into a finite direct sum of objects having local endomorphism rings [1, 2, 3, 7, 13. This in particular implies that any object $X \in \mathcal{B}$ can be decomposed into a finite direct sum of indecomposable objects, and the collection of indecomposable summands is determined uniquely up to isomorphism. If $\mathcal{B}$ is linear over a field, Karoubi complete, and has finite-dimensional morphism spaces, then $\mathcal{B}$ is a Krull-Schmidt category.

For any additive category $\mathcal{B}$, we denote by $\overline{\mathcal{B}}$ its Karoubi completion. We will need the following standard facts.

Proposition 2.1. 1) For a small additive Krull-Schmidt category $\mathcal{B}$, we have a natural isomorphism $K_{0}(\mathcal{B}) \cong \mathbb{Z}^{(S)}$, where $S$ is the set of isomorphism classes of indecomposable objects in $\mathcal{B}$, and $\mathbb{Z}^{(S)}$ denotes the free abelian group generated by $S$. In particular, for $X, Y \in \mathcal{B}$, the equality $[X]=[Y]$ in $K_{0}(\mathcal{B})$ implies $X \cong Y$.

2) For any small additive category $\mathcal{B}$, the natural morphism $K_{0}(\mathcal{B}) \rightarrow K_{0}(\overline{\mathcal{B}})$ is injective.

Proof. Part 1) follows immediately from the unique decomposition into indecomposables.

Part 2) is proved as follows. Suppose that $X, Y \in \mathcal{B}$, and $[X]-[Y] \in K_{0}(\mathcal{B})$ is mapped to zero in $K_{0}(\overline{\mathcal{B}})$. Then there exists an object $Z \in \overline{\mathcal{B}}$ such that $X \oplus Z \cong Y \oplus Z$. Let us choose $Z^{\prime} \in \overline{\mathcal{B}}$, such that $Z \oplus Z^{\prime}$ is contained in the essential image of $\mathcal{B}$. Then we have $X \oplus\left(Z \oplus Z^{\prime}\right) \cong Y \oplus\left(Z \oplus Z^{\prime}\right)$, hence $X$ and $Y$ have the same class in $K_{0}(\mathcal{B})$.

From now on in this section we assume that $\mathcal{A}$ is a small additive category, such that the abelian groups of morphisms $\mathcal{A}(X, Y)$ are finitely generated. By the above discussion, for any prime number $p$, the Karoubi completion $\overline{\mathcal{A}_{\mathbb{F}_{p}}}$ is a Krull-Schmidt category. 
Lemma 2.2. Suppose that $X, Y \in \mathcal{A}$ are two objects such that $[X]=[Y]$ in $K_{0}(\mathcal{A})$. Then $X$ is a retract of $Y^{n}$ for some $n>0$.

Proof. Let us denote by $I \subset \operatorname{End}(X)$ the abelian subgroup generated by the compositions $X \rightarrow Y \rightarrow X$. Clearly, $I$ is a two-sided ideal in $\operatorname{End}(X)$. It suffices to prove that $I=$ $\operatorname{End}(X)$. Indeed, if this is the case, then for some $n>0$ we can find the morphisms $f_{1}, \ldots, f_{n}: X \rightarrow Y$, and $g_{1}, \ldots, g_{n}: Y \rightarrow X$ such that $\sum_{i=1}^{n} g_{i} f_{i}=\mathbf{1}_{X}$. Then the n-tuple $\left(f_{1}, \ldots, f_{n}\right)$ (resp. $\left.\left(g_{1}, \ldots, g_{n}\right)\right)$ defines a morphism $f: X \rightarrow Y^{n}\left(\right.$ resp. $\left.g: Y^{n} \rightarrow X\right)$, such that $g f=\mathbf{1}_{X}$.

Now assume the contrary: the inclusion $I \subset \operatorname{End}(X)$ is strict. Then we can find a prime number $p$ such that $p$ acts non-trivially on the quotient $\operatorname{End}(X) / I$. Let us denote by $\operatorname{pr}: \mathcal{A} \rightarrow \mathcal{A}_{\mathbb{F}_{p}}$ the natural functor. The compositions $\operatorname{pr}(X) \rightarrow \operatorname{pr}(Y) \rightarrow \operatorname{pr}(X)$ generate the ideal $I^{\prime} \subset \operatorname{End}(\operatorname{pr}(X))$, which equals $\operatorname{Im}\left(I \otimes \mathbb{F}_{p} \rightarrow \operatorname{End}(X) \otimes \mathbb{F}_{p}\right)$. By our assumptions on $p$ and $I$, the inclusion $I^{\prime} \subset \operatorname{End}(\operatorname{pr}(X))$ is strict. In particular, the objects $\operatorname{pr}(X)$ and $\operatorname{pr}(Y)$ are non-isomorphic. Since the Karoubi closure $\overline{\mathcal{A}_{\mathbb{F}_{p}}}$ is Krull-Schmidt, we deduce from Proposition 2.1 that $[\operatorname{pr}(X)] \neq[\operatorname{pr}(Y)]$ in $K_{0}\left(\mathcal{A}_{\mathbb{F}_{p}}\right)$, which contradicts the equality $[X]=[Y]$.

For an arbitrary ring $R$, we denote by $K_{+}(R)$ the monoid of isomorphism classes of finitely generated projective right R-modules. If $R$ is noetherian, we denote by $K_{0}^{\prime}(R)$ the Grothendieck group of the abelian category $\bmod -R$ of finitely generated right $R$ modules.

Theorem 2.3. Suppose that $X \in \mathcal{A}$ is an object such that the map $K_{+}(\operatorname{End}(X)) \rightarrow$ $K_{0}^{\prime}(\operatorname{End}(X))$ is injective (for example, this holds if $\operatorname{End}(X) \cong \mathbb{Z}$, since $K_{+}(\mathbb{Z})=\mathbb{Z}_{\geq 0}$ clearly injects into $\left.K_{0}^{\prime}(\mathbb{Z})=\mathbb{Z}\right)$. Then for any object $Y \in \mathcal{A}$ such that $[X]=[Y]$ we have $X \cong Y$.

Proof. By Proposition 2.1 2), we may and will assume that $\mathcal{A}$ is Karoubi complete. Let us denote by $\operatorname{add}(X) \subset \mathcal{A}$ the full subcategory consisting of direct summands of direct sums of copies of $X$. Also, we put $R:=\operatorname{End}(X)$, and denote by $\operatorname{Proj}_{f . g}-R$ the category of finitely generated projective right $R$-modules.

Then the functor $\operatorname{Hom}(X,-): \mathcal{A} \rightarrow \bmod -R$ restricts to an equivalence $\operatorname{add}(X) \stackrel{\sim}{\rightarrow}$ $\operatorname{Proj}_{f . g .}-R$. Now take some $Y \in \mathcal{A}$ such that $[X]=[Y]$. Applying the functor $\operatorname{Hom}(X,-)$ to the objects $X$ and $Y$, we see that $[R]=[\operatorname{Hom}(X, Y)]$ in $K_{0}^{\prime}(R)$ (in fact the classes of $R$ and $\operatorname{Hom}(X, Y)$ are already equal in the Grothendieck group of $\bmod -R$ as an additive category, hence also in $\left.K_{0}^{\prime}(R)\right)$. Applying Lemma2.2 to $X$ and $Y$ (with roles inversed), we obtain $Y \in \operatorname{add}(X)$, hence $\operatorname{Hom}(X, Y) \in \operatorname{Proj}_{f . g}-R$. This in turn yields the isomorphism 
of $R$-modules $R \cong \operatorname{Hom}(X, Y)$, since the map $K_{+}(R) \rightarrow K_{0}^{\prime}(R)$ is injective. Finally, since $Y \in \operatorname{add}(X)$, by applying a quasi-inverse equivalence $(\operatorname{Hom}(X,-))^{-1}: \operatorname{Proj}_{f . g .}-R \stackrel{\sim}{\rightarrow}$ $\operatorname{add}(X)$ we conclude that $X \cong Y$ in $\mathcal{A}$.

In this paper we apply Theorem 2.3 only to the cases when $\operatorname{End}(X) \cong \mathbb{Z}$.

We now turn to showing that each stable isomorphism class of objects in $\mathcal{A}$ contains only finitely many isomorphism classes. For any unital ring $R$, we denote by $R^{\times}$the group of invertible elements in $R$.

Lemma 2.4. Let $\varphi: A \rightarrow B$ be a surjective homomorphism of artinian rings. Then the group homomorphism $\varphi^{\times}: A^{\times} \rightarrow B^{\times}$is surjective.

Proof. First let us assume that $A$ is semi-simple. Then $A$ is isomorphic to the direct product of simple artinian rings (which are matrix algebras over skew-fields): $A \cong \prod_{i=1}^{k} A_{i}$. The ideal $\operatorname{ker}(\varphi)$ is isomorphic to a direct product of some of the $A_{i}$ 's: $\operatorname{ker}(\varphi)=\prod_{i \in M}^{i=1} A_{i}$, $M \subseteq\{1, \ldots, k\}$. Hence $B \cong \prod_{\substack{1 \leq i \leq k \\ i \notin M}} A_{i}$ is also semi-simple and we have a surjection

$$
A^{\times} \cong \prod_{i=1}^{k} A_{i}^{\times} \rightarrow \prod_{\substack{1 \leq i \leq k \\ i \notin M}} A_{i}^{\times} \cong B^{\times}
$$

Now we consider the general case. Denote by $J_{A} \subset A, J_{B} \subset B$ the Jacobson radicals. Since $J_{A}$ consists of nilpotent elements and $\varphi\left(J_{A}\right) \subset B$ is an ideal, we have $\varphi\left(J_{A}\right) \subseteq J_{B}$. On the other hand, since $B / \varphi\left(J_{A}\right)$ is semi-simple (by the above discussion), we see that $\varphi\left(J_{A}\right)=J_{B}$. Note that an element of $A$ is invertible if and only if its image in $A / J_{A}$ is invertible.

Now take any $x \in B^{\times}$. We can choose some $y \in A^{\times}$such that $\varphi(y) \in x+J_{B}$. Finally, we can choose some $z \in J_{A}$ such that $\varphi(z)=x-\varphi(y)$. Then $y+z \in A^{\times}$and $\varphi(y+z)=x$. This proves the lemma.

Lemma 2.5. Let $f: R \rightarrow S$ be a surjective homomorphism of unital rings such that

(i) the ring $R$ is a finitely generated abelian group;

(ii) the ideal $I=\operatorname{ker}(f)$ is finite;

(iii) $f: R \rightarrow S$ is a split surjection in the category of abelian groups.

Then the induced group homomorphism $f^{\times}: R^{\times} \rightarrow S^{\times}$is surjective.

Proof. We assume that $I \neq 0$, otherwise there is nothing to prove. Let us put $N:=|I|$, so that $N I=0$. Choosing a splitting which exists by (iii), we get an isomorphism of abelian groups $R \cong I \oplus S$. It induces an isomorphism $R / N R \cong I \oplus S / N S$. It follows that we have an 
isomorphism of rings $R \rightarrow R / N R \times_{S / N S} S$. This in turn implies an isomorphism of groups $R^{\times} \rightarrow(R / N R)^{\times} \times(S / N S)^{\times} S^{\times}$. By (i), the ring $R / N R$ is artinian. By Lemma 2.4, the group homomorphism $(R / N R)^{\times} \rightarrow(S / N S)^{\times}$is surjective. Therefore, the homomorphism $f^{\times}: R^{\times} \rightarrow S^{\times}$is also surjective.

For any ring $A$, we say that a right $A$-module is $n$-generated if it can be generated by $n$ elements.

Proposition 2.6. Let $A$ be a unital ring which is a finitely generated abelian group. Then for any $n>0$ there are only finitely many isomorphism classes of $n$-generated right projective $A$-modules.

Proof. Replacing $A$ by the matrix ring $M_{n}(A)$ (via Morita equivalence), we reduce to the case $n=1$.

First we consider the case when $A$ is torsion free over $\mathbb{Z}$, so that $A \subset A_{\mathbb{Q}}$ is an order. Let us denote by $I \subset A_{\mathbb{Q}}$ the Jacobson radical, and put $J:=I \cap A$. Then $A / J$ is an order in a semi-simple algebra $A_{\mathbb{Q}} / I$, so by Jordan-Zassenhaus theorem [18, Theorem 26.4] there are only finitely many isomorphism classes of $(A / J)$-modules which are torsion free finitely generated over $\mathbb{Z}$ of rank at most $\operatorname{dim}\left(A_{\mathbb{Q}} / I\right)$. Thus, there are only finitely many isomorphism classes of projective cyclic $(A / J)$-modules. Finally, these are in bijection with isomorphism classes of projective cyclic $A$-modules, since $J$ is nilpotent.

Now let us consider the general case. Let us denote by $A_{\text {tors }} \subset A$ the ideal of $\mathbb{Z}$-torsion elements, and put $B:=A / A_{\text {tors }}$. Recall that two idempotents $e, e^{\prime} \in B$ are conjugate if and only if there are isomorphisms of right $B$-modules $e B \cong e^{\prime} B,(1-e) B \cong\left(1-e^{\prime}\right) B$. Since $B$ is torsion free, we already know that there are only finitely many idempotents in $B$ up to conjugation.

By Lemma 2.5, the homomorphism $A^{\times} \rightarrow B^{\times}$is surjective. It follows that there are only finitely many idempotents in $A$ up to conjugation, since the fibers of the map of sets $A \rightarrow B$ are finite of cardinality $\left|A_{\text {tors }}\right|$. This proves the proposition.

Theorem 2.7. For any object $X$ in $\mathcal{A}$, there are only finitely many isomorphism classes of objects $Y \in \mathcal{A}$ such that $[X]=[Y]$ in $K_{0}(\mathcal{A})$.

Proof. By Lemma 2.2 and its proof, for any $Y \in \mathcal{A}$ with $[X]=[Y], X$ is a retract of $Y^{n}$, where $n$ is the smallest number of generators of the right $\operatorname{End}_{\mathcal{A}}(X)$-module $\operatorname{Hom}_{\mathcal{A}}(X, Y)$. By Proposition 2.6, the number of isomorphism classes of retracts of $Y^{n}$ is finite for a given $n$. Thus, it suffices to find an upper bound for $n$.

Since $X$ and $Y$ become isomorphic in $\mathcal{A}_{\mathbb{F}_{p}}$ for all primes $p$, we have isomorphisms $\operatorname{End}_{\mathcal{A}}(X) \otimes \mathbb{F}_{p} \cong \operatorname{Hom}_{\mathcal{A}}(X, Y) \otimes \mathbb{F}_{p}$. Now for any finitely generated abelian group $M$, its 
smallest number of generators equals $\max _{p}\left(\operatorname{dim}_{\mathbb{F}_{p}}\left(M \otimes_{\mathbb{Z}} \mathbb{F}_{p}\right)\right)$. We conclude that the abelian groups $\operatorname{End}_{\mathcal{A}}(X)$ and $\operatorname{Hom}_{\mathcal{A}}(X, Y)$ have the same smallest numbers of generators, say $m$. In particular, $m$ does not depend on $Y$. The abelian group $\operatorname{Hom}_{\mathcal{A}}(X, Y)$ is therefore $m$-generated as a right $\operatorname{End}_{\mathcal{A}}(X)$-module. This proves the theorem.

\section{L-EQUivalenCE of ALGEBRAIC VARIETIES}

By the famous result of Looijenga and Bittner [15, 4, there is an alternative description of $K_{0}\left(\operatorname{Var}_{\mathbb{C}}\right)$ : it is generated by isomorphism classes of smooth projective complex algebraic varieties, subject to relation $[X]+[Z]=[Y]+[E]$, whenever $Y$ is smooth projective, $Z \subset Y$ is a smooth subvariety, $X$ is a blow-up of $Y$ along $Z$, and $E \subset X$ is the exceptional divisor.

For each integer $n \in \mathbb{Z}$, we denote by $\mathrm{HS}_{\mathbb{Z}, n}$ the additive category of pure integral polarizable Hodge structures of weight $n$. We also denote by $\mathrm{HS}_{\mathbb{Z}}:=\bigoplus_{n \in \mathbb{Z}} \mathrm{HS}_{\mathbb{Z}, n}$ the additive category of graded pure polarizable Hodge structures. We denote by $K_{0}^{a d d}\left(\mathrm{HS}_{\mathbb{Z}}\right)$ the Grothendieck group of the additive category $\mathrm{HS}_{\mathbb{Z}}$. Of course, we have a direct sum decomposition $K_{0}^{a d d}\left(\mathrm{HS}_{\mathbb{Z}}\right)=\bigoplus_{n \in \mathbb{Z}} K_{0}^{a d d}\left(\mathrm{HS}_{\mathbb{Z}, n}\right)$.

We claim that there is a natural homomorphism of abelian groups $\operatorname{Hdg}_{\mathbb{Z}}: K_{0}\left(\operatorname{Var}_{\mathbb{C}}\right) \rightarrow$ $K_{0}^{a d d}(\mathrm{HS})$, given by $\operatorname{Hdg}_{\mathbb{Z}}([X])=\left[H^{\bullet}(X)\right]$ for a smooth projective $X$. Indeed, within the above notation for $X, Y, Z$ and $E$, we have natural isomorphisms of graded pure Hodge structures

$$
H^{\bullet}(E) \cong H^{\bullet}(Z) \oplus H^{\bullet}(Z)(-1) \oplus \cdots \oplus H^{\bullet}(Z)(1-c),
$$

and

$$
H^{\bullet}(X) \cong H^{\bullet}(Y) \oplus H^{\bullet}(Z)(-1) \oplus \cdots \oplus H^{\bullet}(Z)(1-c),
$$

where $c=\operatorname{codim}_{Y} Z$ (see [16]). Hence, $\operatorname{Hdg}_{\mathbb{Z}}([X])+\operatorname{Hdg}_{\mathbb{Z}}([Z])=\operatorname{Hdg}_{\mathbb{Z}}([Y])+\operatorname{Hdg}_{\mathbb{Z}}([E])$, which means that the homomorphism $\mathrm{Hdg}_{\mathbb{Z}}$ is well-defined.

Next, we observe that for a smooth projective $X$ we have $\operatorname{Hdg}_{\mathbb{Z}}([X] \cdot \mathbb{L})=$ $\operatorname{Hdg}_{\mathbb{Z}}([X])(-1)$. This follows immediately from the equality $[X] \cdot \mathbb{L}=\left[X \times \mathbb{P}^{1}\right]-[X]$, and the isomorphism $H^{\bullet}\left(X \times \mathbb{P}^{1}\right) \cong H^{\bullet}(X) \oplus H^{\bullet}(X)(-1)$. Since the Tate twist $-(-1)$ is an auto-equivalence of $\mathrm{HS}_{\mathbb{Z}}$, we can factor the homomorphism $\operatorname{Hdg}_{\mathbb{Z}}$ through $K_{0}\left(\operatorname{Var}_{\mathbb{C}}\right)\left[\mathbb{L}^{-1}\right]$ via $\operatorname{Hdg}_{\mathbb{Z}}\left([X] \cdot \mathbb{L}^{-n}\right)=\left[H^{\bullet}(X)(n)\right]$.

We now use the resulting homomorphism

$$
\operatorname{Hdg}_{\mathbb{Z}}: K_{0}\left(\operatorname{Var}_{\mathbb{C}}\right)\left[\mathbb{L}^{-1}\right] \rightarrow K_{0}^{a d d}\left(\mathrm{HS}_{\mathbb{Z}}\right)
$$

to distinguish L-equivalence classes of varieties. The following result provides counterexamples to Conjecture 1.1. These counterexamples appeared independently in [12, Corollary 7.4, Lemma 7.6]. 
Theorem 3.1. Let $A$ be an abelian variety such that $\operatorname{End}(A)=\mathbb{Z}$. Then

1) for any abelian variety $A^{\prime}$ such that $\left[H^{1}(A)\right]=\left[H^{1}\left(A^{\prime}\right)\right]$ in $K_{0}^{\text {add }}\left(\mathrm{HS}_{\mathbb{Z}, 1}\right)$ we have $A \cong A^{\prime}$.

2) for any abelian variety $A^{\prime}$, which is L-equivalent to $A$, we have $A \cong A^{\prime}$. In particular, if $A$ is not principally polarizable, then $A$ is not L-equivalent to $\hat{A}$, but $D^{b}(A) \cong D^{b}(\hat{A})$. In particular, D-equivalence does not imply L-equivalence.

Proof. We observe that $\operatorname{End}_{\mathrm{HS}_{\mathbb{Z}, 1}}\left(H^{1}(A)\right)=\operatorname{End}(A)^{o p}=\mathbb{Z}$. Hence, part 1) follows directly from Theorem 2.3 .

Part 2) follows from part 1) by applying the composition

$$
\operatorname{Hdg}_{\mathbb{Z}}: K_{0}\left(\operatorname{Var}_{\mathbb{C}}\right)\left[\mathbb{L}^{-1}\right] \rightarrow K_{0}^{a d d}\left(\mathrm{HS}_{\mathbb{Z}}\right) \rightarrow K_{0}^{a d d}\left(\mathrm{HS}_{\mathbb{Z}, 1}\right) .
$$

Theorem 3.2. There are only finitely many abelian varieties in each L-equivalence class.

Proof. This follows immediately from the homomorphism (3.1) and Theorem 2.7, since an abelian variety is reconstructed from the integral Hodge structure on $H^{1}$.

We now consider K3 surfaces.

For a $\mathrm{K} 3$ surface $X$, we denote by $T(X) \subset H^{2}(X, \mathbb{Z})$ the integral Hodge substructure of transcendental cycles. The sublattice $T(X) \oplus N S(X) \subset H^{2}(X, \mathbb{Z})$ has finite index, say $D$, which is also equal to the determinant of the restriction of the intersection form to $T(X)$.

Proposition 3.3. Given a K3 surface $X$, there are only finitely many isomorphism classes of $K 3$ surfaces $X^{\prime}$ such that the Hodge structures $H^{2}(X, \mathbb{Z})$ and $H^{2}\left(X^{\prime}, \mathbb{Z}\right)$ are isomorphic (but not necessarily isometric).

Proof. An isomorphism of Hodge structures $H^{2}(X, \mathbb{Z}) \stackrel{\sim}{\rightarrow} H^{2}\left(X^{\prime}, \mathbb{Z}\right)$ implies an isomorphism of sublattices $T(X) \oplus N S(X) \stackrel{\sim}{\rightarrow} T\left(X^{\prime}\right) \oplus N S\left(X^{\prime}\right)$, and an isomorphism of Hodge structures $T(X)$ and $T\left(X^{\prime}\right)$. In particular, the intersection forms restricted to $T(X)$ and $T\left(X^{\prime}\right)$ have the same determinant $D$.

Let us put $R:=\operatorname{End}_{\mathrm{HS}_{\mathbb{Z}, 2}}(T(X))$, and $G:=R^{\times}=\operatorname{Aut}_{\mathrm{HS}_{\mathbb{Z}, 2}}(T(X))$. By [17, Theorem 3.3 ] and [5, Proposition 5.3], there are only finitely many non-isomorphic K3 surfaces $X^{\prime}$ with a Hodge isometry $T(X) \cong T\left(X^{\prime}\right)$. Therefore, it suffices to show that there are only finitely many $G$-orbits in the set $S_{D}$ of all symmetric pairings $T(X) \otimes T(X) \rightarrow \mathbb{Z}(-2)$ with determinant $D$ (the pairing is required to be a morphism in $\mathrm{HS}_{\mathbb{Z}, 4}$ ).

By Zarhin's theorem [20], the $\mathbb{Q}$-algebra $R_{\mathbb{Q}}$ is either a totally real number field $F$, or an imaginary quadratic extension $E$ of a totally real number field, which we also denote by $F$. In both cases the complex conjugation gives a well-defined involution $\sigma: R_{\mathbb{Q}} \rightarrow R_{\mathbb{Q}}$, 
which restricts to an involution on $R$. We put $R^{\prime}:=R^{\sigma}=R \cap F$. For any $a \in R_{\mathbb{Q}}$, and $x, y \in T(X)$, we have $\langle a(x), y\rangle=\langle x, \sigma(a)(y)\rangle$, where $\langle$,$\rangle denotes the intersection form.$

Now let $\beta$ denote any symmetric Hodge pairing on $T(X)$ of determinant $D$. It defines an element $a(\beta) \in F$ such that $\beta(x, y)=\langle a(\beta)(x), y\rangle$. Let us denote by $M_{D}$ the set of $a \in F$ such that both $D a$ and $D a^{-1}$ are in $R^{\prime}$. Clearly, we have $a(\beta) \in M_{D}$. Let us note that $\beta_{1}$ and $\beta_{2}$ are in the same $G$-orbit if and only if there exists $b \in R^{\times}$such that $a\left(\beta_{1}\right)=b \sigma(b) a\left(\beta_{2}\right)$.

It suffices to show that there are only finitely many $\left(R^{\prime \times}\right)^{2}$-orbits in $M_{D}$, where the action is given by multiplication. Since $R^{\prime}$ is an order in $F$, we have $R^{\prime} \subset \mathcal{O}_{F}$, and the group $R^{\prime} \times$ is a finite index subgroup of $\mathcal{O}_{F}^{\times}$. Since we are proving the assertion about $R^{\prime}$ (and not about $X$ ), we may and will assume that $R^{\prime}=\mathcal{O}_{F}$. By the Dirichlet Unit Theorem, the group $\mathcal{O}_{F}^{\times}$is finitely generated, hence its subgroup $\left(\mathcal{O}_{F}^{\times}\right)^{2}$ has finite index. It remains to note that the set $M_{D}$ has only finitely many $\mathcal{O}_{F}^{\times}$-orbits. Indeed, for any $a \in M_{D}$ and any maximal ideal $\rho \subset \mathcal{O}_{F}$, we have $\left|\operatorname{ord}_{\rho}(a)\right| \leq \operatorname{ord}_{\rho}(D)$, hence $\left|M_{D} / \mathcal{O}_{F}^{\times}\right| \leq$ $\prod_{\rho \ni D}\left(2 \operatorname{ord}_{\rho}(D)+1\right)$. This proves the proposition.

Theorem 3.4. For any $K 3$ surface $X$, there are only finitely many isomorphism classes of K3 surfaces $Y$ which are L-equivalent to $X$.

Proof. By Theorem 2.7, there are only finitely many isomorphism classes of possible Hodge structures $H^{2}(Y, \mathbb{Z})$ for $\mathrm{K} 3$ surfaces $Y$ that are L-equivalent to $X$. By Proposition 3.3 , there are only finitely many isomorphism classes of $\mathrm{K} 3$ surfaces with a given (isomorphism class of) integral Hodge structure on $H^{2}$. This proves the theorem.

Now we disprove Conjecture 1.2 .

Corollary 3.5. There are isogenous Kummer surfaces which are not L-equivalent.

Proof. By [8, Theorem 0.1], for any Kummer surface $X$ there are only finitely many isomorphism classes of abelian surfaces $A$ such that $X \cong \mathrm{Km} A$. Thus, there are countably many Kummer surfaces in each isogeny class. On the other hand, by Theorem 3.4 there are only finitely many Kummer surfaces in each L-equivalence class. This proves the assertion.

Finally, we show that there are derived equivalent twisted K3 surfaces such that the underlying K3 surfaces are not L-equivalent. Recall that for a smooth complex projective variety $X$ its Brauer group $\operatorname{Br}(X)$ is the torsion part of $H_{a n}^{2}\left(X, \mathcal{O}_{X}^{\times}\right)$. For any element $\alpha \in \operatorname{Br}(X)$ we denote by $\operatorname{Coh}(X, \alpha)$ the abelian category of $\alpha$-twisted coherent sheaves [6], and by $D^{b}(X, \alpha)$ its derived category. By definition, a twisted K3 surface is a pair $(X, \alpha)$, where $X$ is a $\mathrm{K} 3$ surface, and $\alpha \in \operatorname{Br}(X)$ is an element. In particular, for any $\mathrm{K} 3$ surface $X$ we have a twisted $\mathrm{K} 3$ surface $(X, 1)$. 
Corollary 3.6. There exist derived equivalent twisted K3 surfaces such that the underlying K3 surfaces are not L-equivalent.

Proof. By Corollary 3.5, there exist isogenous Kummer surfaces $S$ and $S^{\prime}$ that are not L-equivalent. By [9, Theorem 0.1] there exist a finite sequence of K3 surfaces $S=S_{0}, S_{1}, \ldots, S_{n}=S^{\prime}$, and Brauer classes $\alpha \in \operatorname{Br}(S), \alpha_{1}, \beta_{1} \in \operatorname{Br}\left(S_{1}\right), \ldots, \alpha_{n-1}, \beta_{n-1} \in$ $\operatorname{Br}\left(S_{n-1}\right), \quad \alpha^{\prime} \in \operatorname{Br}\left(S^{\prime}\right)$, and derived equivalences $D^{b}\left(S_{0}, \alpha\right) \simeq D^{b}\left(S_{1}, \alpha_{1}\right), \quad D^{b}\left(S_{1}, \beta_{1}\right) \simeq$ $D^{b}\left(S_{2}, \alpha_{2}\right), \ldots, D^{b}\left(S_{n-1}, \beta_{n-1}\right) \simeq D^{b}\left(S_{n}, \alpha^{\prime}\right)$. Since $S$ and $S^{\prime}$ are not L-equivalent, we have that for some $i \in\{0,1 \ldots, n-1\}, S_{i}$ and $S_{i+1}$ are not L-equivalent. This proves the assertion.

For completeness we also present more explicitly a large class of examples of non-Lequivalence for twisted derived equivalent K3 surfaces. Recall that for any K3 surface $X$ we have a natural isomorphism of abelian groups $\operatorname{Br}(X) \cong \operatorname{Hom}_{\mathbb{Z}}(T(X), \mathbb{Q} / \mathbb{Z})$, see e.g. [19, Section 4.1]. In particular, the elements of order $n$ in $\operatorname{Br}(X)$ correspond to surjective homomorphisms $T(X) \rightarrow \mathbb{Z} / n \mathbb{Z}$.

Lemma 3.7. If the K3 surfaces $X$ and $X^{\prime}$ are L-equivalent, then we have $[T(X)]=$ $\left[T\left(X^{\prime}\right)\right]$ in $K_{0}^{\text {add }}\left(\mathrm{HS}_{\mathbb{Z}, 2}\right)$. If moreover we have $\operatorname{End}_{\mathrm{HS}_{\mathbb{Z}, 2}}(T(X))=\mathbb{Z}$, then $T(X) \cong T\left(X^{\prime}\right)$ in $\mathrm{HS}_{\mathbb{Z}, 2}$.

Proof. The first statement follows from the equality $\left[H^{2}(X, \mathbb{Z})\right]=\left[H^{2}\left(X^{\prime}, \mathbb{Z}\right)\right]$, and from the existence of an additive endofunctor $T: \mathrm{HS}_{\mathbb{Z}} \rightarrow \mathrm{HS}_{\mathbb{Z}}$ which assigns to each polarizable pure integral Hodge structure its transcendental Hodge substructure. The second statement follows from Theorem 2.3 .

Proposition 3.8. Let $X$ be a K3 surface such that $\rho(X) \geq 12$ and $\operatorname{End}_{\mathrm{HS}_{\mathbb{Z}, 2}}(T(X))=\mathbb{Z}$. Take any non-trivial Brauer class $\alpha \in \operatorname{Br}(X)$. Then there exists a K3 surface $Z$ such that $D^{b}(Z, 1) \simeq D^{b}(X, \alpha)$, but $X$ and $Z$ are not L-equivalent.

Proof. Existence of $Z$ with required derived equivalence follows from [11, Proposition 7.3]. By the construction in loc. cit. and by [10, Proposition 4.7], we have an isomorphism of integral Hodge structures $T(Z) \cong T(X, \alpha)$, where $T(X, \alpha)=\operatorname{ker}(\alpha: T(X) \rightarrow \mathbb{Z} / n \mathbb{Z})$, and $n>1$ is the order of $\alpha$. By our assumption on the endomorphisms of $T(X)$ (and since $\mathrm{rk}_{\mathbb{Z}} T(X) \geq 2$ ), the integral Hodge structures $T(X)$ and $T(Z)$ are non-isomorphic. Hence, by Lemma 3.7 $X$ and $Z$ are not L-equivalent.

Remark 3.9. We note that if one considers further localization of $K_{0}\left(\operatorname{Var}_{\mathbb{C}}\right)\left[\mathbb{L}^{-1}\right]$ with respect to some set $W \subset \mathbb{Z}[\mathbb{L}]$ of monic polynomials, then equality $[X]=[Y]$ in $K_{0}\left(\operatorname{Var}_{\mathbb{C}}\right)\left[\mathbb{L}^{-1}\right]\left[W^{-1}\right]$ would also imply $\operatorname{Hdg}_{\mathbb{Z}}([X])=\operatorname{Hdg}_{\mathbb{Z}}([Y])$. This is because there are 
no non-zero $\operatorname{Hdg}_{\mathbb{Z}}(W)$-torsion elements in $K_{0}^{\text {add }}\left(\mathrm{HS}_{\mathbb{Z}}\right)$. In particular, all our statements concerning (non-)L-equivalence are also valid in such further localizations.

\section{REFERENCES}

[1] M. Atiyah, On the Krull-Schmidt theorem with application to sheaves, Bull. Soc. Math. France 84 (1956) 307-317.

[2] M. Auslander, Representation Dimension of Artin Algebras, in: Queen Mary College Mathematics Notes, London, 1971.

[3] M. Auslander, Representation theory of Artin algebras. I, II, Comm. Algebra 1 (1974) 177-268; ibid. 1 (1974), 269-310.

[4] F. Bittner, The universal Euler characteristic for varieties of characteristic zero. Compositio Mathematica, 140(4), 1011-1032.

[5] T. Bridgeland, A. Maciocia, Complex surfaces with equivalent derived categories, Math. Z. 236 (2001), 677-697.

[6] A. Căldăraru. Derived categories of twisted sheaves on Calabi-Yau manifolds. Ph.-D. thesis Cornell, 2000.

[7] P. Gabriel, A.V. Roiter, Representations of Finite-dimensional Algebras, Translated from the Russian, reprint of the 1992 English translation, Springer, Berlin, 1997.

[8] S. Hosono, B.H. Lian, K. Oguiso, S.-T. Yau, Kummer structures on a K3 surface - An old question of T. Shioda, Duke Math. J. 12 (2003), 635-647.

[9] D. Huybrechts, Motives of isogenous K3 surfaces, arXiv:1705.04063 (preprint), to appear in Commentarii Mathematici Helvetici.

[10] D. Huybrechts, Generalized Calabi-Yau structures, K3 surfaces, and B-fields. Internat. J. Math. 16 (2005), no. 1, 13-36.

[11] D. Huybrechts, P. Stellari, Equivalences of twisted K3 surfaces. Math. Ann. 332 (2005), no. 4, 901-936.

[12] A. Ito, M. Miura, S. Okawa, K. Ueda, Derived equivalence and Grothendieck ring of varieties: the case of K3 surfaces of degree 12 and abelian varieties, arXiv:1612.08497 (preprint).

[13] H. Krause, Krull-Schmidt categories and projective covers, Expositiones Mathematicae Volume 33, Issue 4, 2015, Pages 535-549.

[14] A. Kuznetsov, E. Schinder, Grothendieck ring of varieties, D- and L-equivalence, and families of quadrics, E. Sel. Math. New Ser. (2017). https://doi.org/10.1007/s00029-017-0344-4.

[15] E. Looijenga, Motivic measures. Séminaire Bourbaki 42 (1999-2000): 267-297.

[16] Yu. I. Manin, Correspondences, motifs and monoidal transformations, Mat. Sb. (N.S.), 77(119):4 (1968), 475-507. English translation: Math. USSR-Sb., 6:4 (1968), 439-470.

[17] D. Orlov, Equivalences of derived categories and K3 surfaces. Algebraic geometry, 7. J. Math. Sci. (New York) 84 (1997), no. 5, 1361-1381.

[18] I. Reiner, "Maximal Orders," Academic Press, London, 1975.

[19] P. Stellari, Derived categories and Kummer varieties. Mathematische Zeitschrift June 2007, Volume 256, Issue 2, pp 425-441.

[20] Y. Zarhin. Hodge groups of K3 surfaces. J. Reine Angew. Math., 341:193-220, 1983.

Steklov Mathematical Institute of RAS, Gubkin str. 8, Moscow 119991, Russia

E-mail address: efimov@mccme.ru 